\title{
Oral $24 \%$ sucrose associated with nonnutritive sucking for pain control in healthy term newborns receiving venipuncture beyond the first week of life
}

This article was published in the following Dove Press journal: Journal of Pain Research

\author{
Giuseppe De Bernardo' \\ Marina Riccitelli \\ Desiree Sordino' \\ Maurizio Giordano 3 \\ Sabrina Piccolo' \\ Giuseppe Buonocore ${ }^{2}$ \\ Serafina Perrone ${ }^{2}$ \\ 'Department of Emergency, NICU, \\ AORN Santobono-Pausilipon, Naples, \\ Italy; ${ }^{2}$ Department of Molecular and \\ Developmental Medicine, University \\ of Siena, Siena, Italy; ${ }^{3}$ Faculty of \\ Medicine, Federico II University, \\ Naples, Italy
}

Objective: To test the hypothesis that oral administration of $24 \%$ sucrose associated with nonnutritive sucking in healthy newborns receiving venipuncture beyond the first week of life controls pain and pain-related variation in heart rate (HR) and noninvasive oxygen saturation $\left(\mathrm{SpO}_{2}\right)$. Methods: A total of 66 term newborns were enrolled between February and September 2017 in the Neonatology Department of AORN Santobono-Pausilipon, Naples. They were randomly assigned to receive oral $1 \mathrm{~mL} 24 \%$ sucrose (treated group [TG], $n=33$; gestational age 38.53 \pm 1.49 weeks; body weight $3,035 \pm 55 \mathrm{~g}$; age $22.40 \pm 6.82$ weeks) or oral $1 \mathrm{~mL} 10 \%$ glucose (control group [CG], $n=33$; gestational age $38.91 \pm 1.45$ weeks; body weight $3,203 \pm 65$ g; age $23.36 \pm 7.02$ weeks) 1 minute before and during venipuncture. Evaluations were carried out between 8 and 9 am in all newborns. The Neonatal Infant Pain Scale (NIPS) was used to assess pain in newborns. Outcome measurements $\left(\mathrm{HR}, \mathrm{SpO}_{2}\right)$ were obtained before (T0), during (T1), and 1 minute after (T2) venipuncture using a Nellcor bedside $\mathrm{SpO}_{2}$ patient-monitoring system. NIPS scores were recorded throughout the procedure. Statistical analysis was performed using SPSS version 20.0. Changes in $\mathrm{HR}$ and $\mathrm{SpO}_{2}$ were assessed by mixed ANOVA for repeated measures. NIPS scores were evaluated by Mann-Whitney $U$ test.

Results: There were no statistically significant differences in $\mathrm{HR}$ or $\mathrm{SpO}_{2}$ between TG and CG at T0. HR was significantly lower in TG than CG at both T1 and T2 $(P<0.05)$, whereas $\mathrm{SpO}_{2}$ was significantly higher in TG than CG at both T1 and T2 $(P<0.05)$. NIPS scores were significantly lower in TG (median 0) than CG (median 6) during the entire procedure $(P<0.05)$.

Conclusion: Oral administration of $24 \%$ sucrose associated with nonnutritive sucking prior to and during a painful procedure has a strong impact on pain response in term newborns, reducing NIPS scores and influencing pain-associated variations in $\mathrm{HR}$ and $\mathrm{SpO}_{2}$. Complete analgesia during painful procedures in term newborns might prevent pain reactivity and its behavioral and neurodevelopmental consequences. Replication of this study is needed before widespread application of findings.

Keywords: oral sucrose, heart rate, neonatal pain

\section{Introduction}

A widely accepted definition of pain was developed by a taxonomy task force of the International Association for the Study of Pain: "Pain is an unpleasant sensory and emotional experience that is associated with actual or potential tissue damage or described in such terms." A key feature of this definition is "pain is always subjective". Pain management in neonates should not just be driven by ethics or empathy,
Correspondence: Serafina Perrone Department of Molecular and

Developmental Medicine, University of Siena, 36 Viale Bracci, Siena 53100, Italy

Tel +390577586542

Fax+39057 7586182

Email saraspv@yahoo.it 
but should be viewed as part of normal medical and nursing care. ${ }^{1}$ Noxious stimulation in the form of venipuncture or heel prick produces a cortical response in both preterm and term newborn infants, measured as an increase in total hemoglobin concentration in the contralateral somatosensory cortex, and nociceptive reflexes, measurable as lower-limb withdrawal and flexor-muscle activity. ${ }^{2}$ Pain is a particular problem for newborns: early in life, it elicits specific behaviors, activates the somatosensory cortex, and stimulates neuroendocrine stress responses, thus leading to short- and long-term clinical consequences. ${ }^{1-4}$

Studies have demonstrated a relationship between pain degree and oxidative stress in healthy full-term newborns at their first pain exposure. We have also reported that sensorial saturation reduces pain score together with pain-related oxidative stress. ${ }^{5}$ Sensorial saturation is a technique used during heel prick in which mothers simultaneously attract their infant's attention by massaging the cheek, speaking to the baby gently, and administering oral glucose. ${ }^{6}$

Multiple trials and systematic reviews have highlighted beneficial analgesic effects of $24 \%$ sucrose for neonates undergoing invasive procedures. ${ }^{7-10}$ Sucrose in combination with other nonpharmacological interventions, such as nonnutritive sucking (NNS) seems to be more effective than sucrose alone. ${ }^{10}$ Nevertheless, there is limited evidence regarding its analgesic efficacy and safety in healthy term newborns beyond the first week of life. In this comparative study, we tested the hypothesis that oral administration of $24 \%$ sucrose associated with NNS in healthy newborns receiving venipuncture beyond the first week of life controls pain and pain-associated variation of heart rate (HR) and noninvasive oxygen saturation $\left(\mathrm{SpO}_{2}\right)$ better that oral administration of $10 \%$ glucose associated with NNS.

\section{Methods}

\section{Participants}

A randomized double-blinded case-control pilot study was conducted from February to September 2017 at the neonatal intensive care unit (NICU) of Santobono-Pausilipon Hospital in Naples, Italy, following research-ethics approval and written informed consent from the families. The study was conducted in accordance with the Declaration of Helsinki. Inclusion criteria were Italian neonates 37-42 weeks gestational age at birth and $>1$ week old at the time of the intervention with body weight $2,500-4,500 \mathrm{~g}$ and able to feed orally. Exclusion criteria were concurrent use of pharmacological pain strategies, neonates with a contraindication for sucrose administration (eg, too ill or unstable for neonatologist assessment, unable to swallow, pharmacologically musclerelaxed), and inability to assess behavioral responses to pain accurately (eg, neonate's face blocked with tape). Patients undergoing painful, stressful procedures or with congenital or perinatal diseases were excluded. Patients born from smoking mothers and those with maternal diabetes, placenta abruption, chronic pathologies, or using drugs/alcohol during pregnancy and infants with fetal growth restriction, fetal malformations, genetic/metabolic disorders, clinical signs of maternal, or fetal infection were also excluded (Figure 1).

Newborns were enrolled after their first week of life during routine control for hyperbilirubinemia or minor surgery in the neonatology ward. Routine controls were carried out in a separate room with 2,000 $1 \mathrm{x}$ between 9 and $10 \mathrm{am} .^{11}$ Another room was used for the treatment intervention and projected to reduce sources of stress, with instrument noise set at $<45 \mathrm{~dB}$ and $10-6001 \mathrm{x} .{ }^{12}$

Newborns were randomly assigned to receive oral sucrose and NNS (treated group ([TG]) or comprised the control group ( $\mathrm{CG}$ ), and randomization was performed using a privacy-protected randomization hospital service. In the $\mathrm{TG}$,

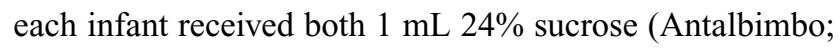
Orsana, Rome, Italy) orally via syringe 1 minute before venipuncture and $1 \mathrm{~mL}$ during the procedure. A pacifier was offered to all neonates immediately following sucrose administration to facilitate NNS, which has been shown to enhance efficacy. ${ }^{10}$ In the $\mathrm{CG}$, each infant received $1 \mathrm{~mL} 10 \%$ glucose orally via syringe with a pacifier 1 minute before venipuncture and during the procedure. Oral solutions were administered drop by drop by an expert caregiver via syringe over the anterior surface of the tongue, allowing for individual neonate swallowing rates over a period of 1 minute. Outcome measures (HR, $\mathrm{SpO}_{2}$, Neonatal Infant Pain Scale [NIPS]) were obtained prior to (T0), during (T1), and 1 minute after venipuncture (T2) in each group. Neonate behavior was scored using the NIPS. Investigators and caregivers were blinded to group assignment.

\section{Instruments}

Data were recorded using a database (Excel 2007) by a pediatric nurse who was aware of the study aims. Parameters collected were sex, gestational age, days of life, body weight, $\mathrm{HR}$ at T0, T1, and $\mathrm{T} 2, \mathrm{SpO}_{2}$ at T0, T1, and T2, and NIPS scores at T1.

Another pediatric nurse aware of the study aims measured the body weight of the newborns with a Seca 354 baby scale and collected NIPS scores after 6 months of NIPS practice. Vital parameters were collected with a Nellcor bedside $\mathrm{SpO}_{2}$ 


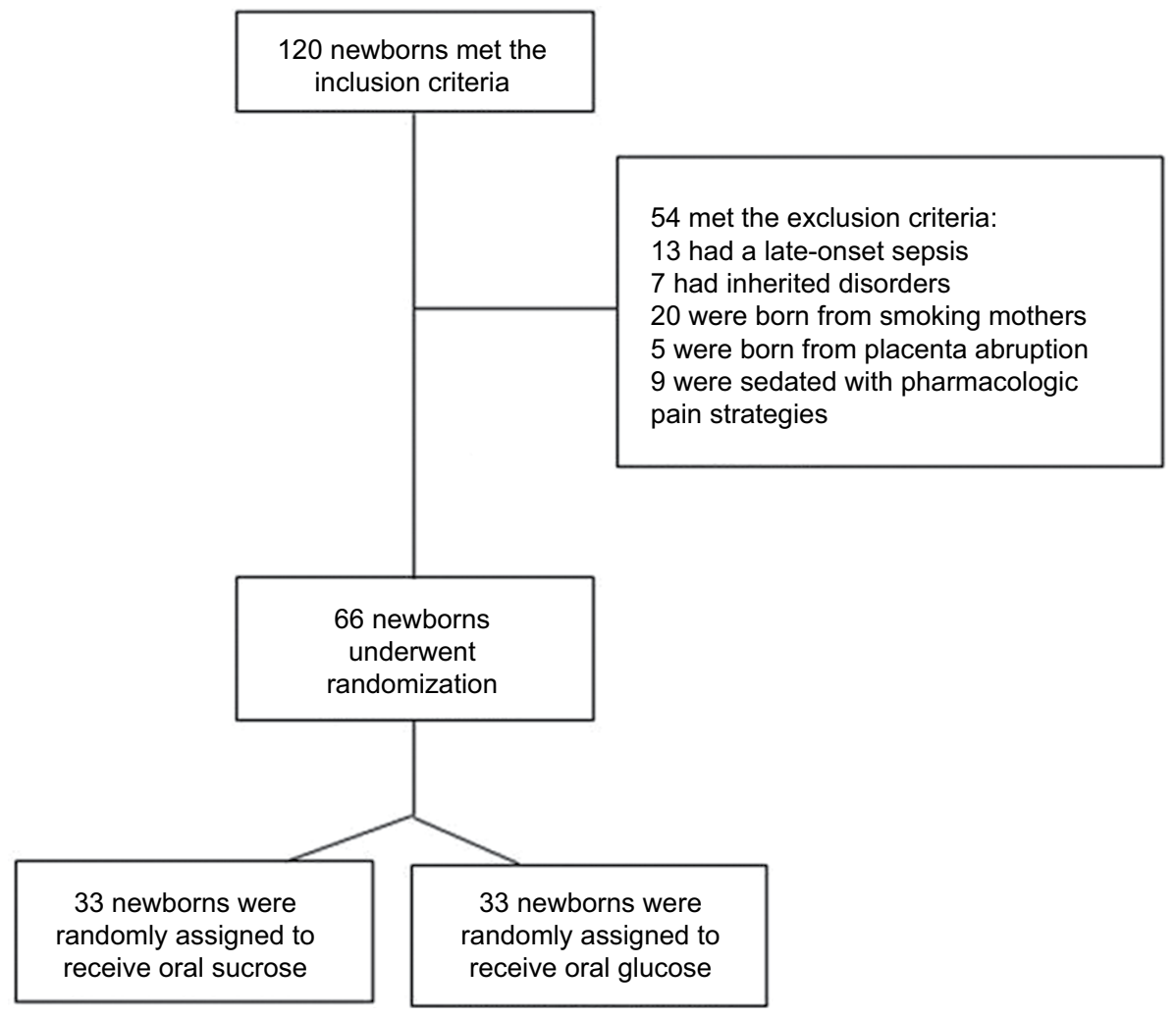

Figure I Enrollment flowchart.

patient-monitoring system. The research nurse placed pulse oximetry probes on the right wrist of every neonate to record $\mathrm{HR}$ and $\mathrm{SpO}_{2}$ continuously and positioned herself in front of the newborn to capture facial movements.

\section{Outcome measures}

The primary outcome was pain intensity measured with the NIPS, a behavioral scale validated by Lawrence et al in $1993^{13}$ that comprises six items (facial expression, cry, breathing, arms, legs, and state of arousal). Caregivers can rate items from 0 (relaxed) to 1 (different than basal) and from 0 (absent) to 2 (vigorous) for cry. A score $\geq 4$ indicates that the newborn feels pain.

The secondary outcome was frequency of a priori-specified adverse event/tolerance criteria (HR $>240$ beats/minute or $\mathrm{HR}<80$ beats/minute for $>20$ seconds, $\mathrm{SpO}_{2}<80 \%$ for $>20$ seconds, no spontaneous respirations for $>20$ seconds, and choking/gagging). Adverse-event data were collected by the research nurse during the intervention, and she also kept a record of "rescue doses" administered (ie, additional doses of sucrose or glucose) if the neonate became overly distressed during the procedure.

\section{Statistical analysis}

Statistical analysis was performed by a statistician who was aware of the study aim using SPSS version 20.0 (IBM, Armonk, NY, USA). Normal distribution of the data was evaluated by Kolmogorov-Smirnov test. Parametric and nonparametric data were evaluated by one-way ANOVA or $\chi^{2}$ test, respectively. Changes in $\mathrm{HR}$ and $\mathrm{SpO}_{2}$ from $\mathrm{T} 0$ to T2 were assessed by mixed ANOVA for repeated measures. $\mathrm{HR}$ and $\mathrm{SpO}_{2}$ were analyzed by reciprocal transformation because of lack of homogeneity. Differences between NIPS scores were evaluated by Mann-Whitney $U$ test. All data with $P<0.05$ were considered significant.

\section{Results}

A total of 66 term newborns were enrolled for the study and randomly assigned to each group (33 in CG and 33 in TG). Demographic characteristics in the two groups were matched and comparable (Table 1). The groups started from similar conditions for $\mathrm{HR}$ and $\mathrm{SpO}_{2}$ at $\mathrm{T} 0$ (Table 2). Changes in vital parameters between groups are reported in Table 2 and Figures 2-4. NIPS scores in both groups were $<4$ at T0; however, scores were significantly lower in TG (range 
1-2 and median 0) compared with $\mathrm{CG}$ at T1 (range 5-7 and median 6). Finally, 100\% of term newborns in CG had a score $\geq 4$ (range 4-6 and median 5) and $100 \%$ of those in TG showed a score $<4$ at T2 (range and median 0 ). There were no reported adverse events defined by a priori criteria among neonates.

Table I Clinical characteristics of study population

\begin{tabular}{|c|c|c|c|}
\hline Clinical characteristics & $\begin{array}{l}\text { Treated } \\
(n=33)\end{array}$ & $\begin{array}{l}\text { Controls } \\
(n=33)\end{array}$ & $P$-value \\
\hline Sex & Male $72 \%$ & Male $67 \%$ & 0.592 \\
\hline Gestational age (weeks) & $38.53 \pm 1.49$ & $38.91 \pm 1.45$ & 0.309 \\
\hline Body weight at birth (g) & $3.035 \pm 0.55$ & $3.203 \pm 0.65$ & 0.267 \\
\hline Days of life & $22.40 \pm 6.82$ & $23.36 \pm 7.02$ & 0.820 \\
\hline Heart rate, T0 & $143 \pm 16.8$ & $147 \pm 12.5$ & 0.181 \\
\hline $\mathrm{SpO}_{2}, \mathrm{TO}$ & $99.48 \pm 0.56$ & $99.57 \pm 0.6 \mathrm{I}$ & 0.538 \\
\hline
\end{tabular}

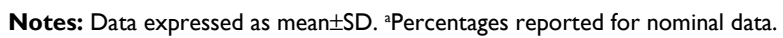

\section{Discussion}

In this study, we report that oral administration of $1 \mathrm{~mL}$ $24 \%$ sucrose prior to and during a single painful procedure appears to be effective in significantly reducing pain in term neonates at the first experience of procedural pain beyond the first week of life. As we know, inadequate pain management in neonatal life is a major impediment to proper neurodevelopment: it alters pain thresholds, physiological responses, and stress- or pain-related behavior beyond early infancy. ${ }^{14}$ Nonpharmacological pain control for infants has been recently supported. Although the impacts of nonpharmacological interventions have been assessed extensively, they were evaluated mostly in sick babies of different postnatal ages using different treatments and pain assessments that yielded ununiform data. Rioualen et al compared sucrose solution or breast-feeding before venipuncture for neonatal screening in healthy 3 -day-old term neonates. ${ }^{15}$ They used

Table 2 Change in vital parameters from T0 (before) to T2 (after venipuncture)

\begin{tabular}{|c|c|c|c|c|c|c|c|}
\hline $\begin{array}{l}\text { Vital } \\
\text { parameters }\end{array}$ & Time & $n$ & Control group & $\begin{array}{l}\text { Treated } \\
\text { group }\end{array}$ & $F(1,60)$ & $P$-value & $R$-value \\
\hline \multicolumn{8}{|l|}{ Heart rate } \\
\hline \multirow[t]{2}{*}{ T0 vs TI } & T0 & 29 & $143 \pm 16.8$ & $147 \pm 12.5$ & \multirow[t]{2}{*}{69.572} & \multirow[t]{2}{*}{$<0.05$} & \multirow[t]{2}{*}{0.73} \\
\hline & TI & 33 & $191.57 \pm 16.94$ & $159.48 \pm 12.46$ & & & \\
\hline \multirow[t]{2}{*}{ TI vs T2 } & TI & 33 & $191.57 \pm 16.94$ & $159.48 \pm 12.46$ & \multirow[t]{2}{*}{16.312} & \multirow[t]{2}{*}{$<0.05$} & \multirow[t]{2}{*}{0.46} \\
\hline & $\mathrm{T} 2$ & 33 & $160.51 \pm 17.65$ & $147.42 \pm 12.05$ & & & \\
\hline \multicolumn{8}{|l|}{$\mathrm{SpO}_{2}$} \\
\hline \multirow[t]{2}{*}{ T0 vs TI } & T0 & 33 & $99.48 \pm 0.56$ & $99.57 \pm 0.61$ & \multirow[t]{2}{*}{215.443} & \multirow[t]{2}{*}{$<0.05$} & \multirow[t]{2}{*}{0.88} \\
\hline & $\mathrm{TI}$ & 33 & $89.93 \pm 4.11$ & $98.39 \pm 0.89$ & & & \\
\hline \multirow[t]{2}{*}{ TI vs T2 } & TI & 33 & $89.93 \pm 4.11$ & $98.39 \pm 0.89$ & \multirow[t]{2}{*}{47.629} & \multirow[t]{2}{*}{$<0.05$} & \multirow[t]{2}{*}{0.66} \\
\hline & T2 & 29 & $97.39 \pm 1.05$ & $99.5 I \pm 0.62$ & & & \\
\hline
\end{tabular}

Notes: Data expressed as mean \pm SD.

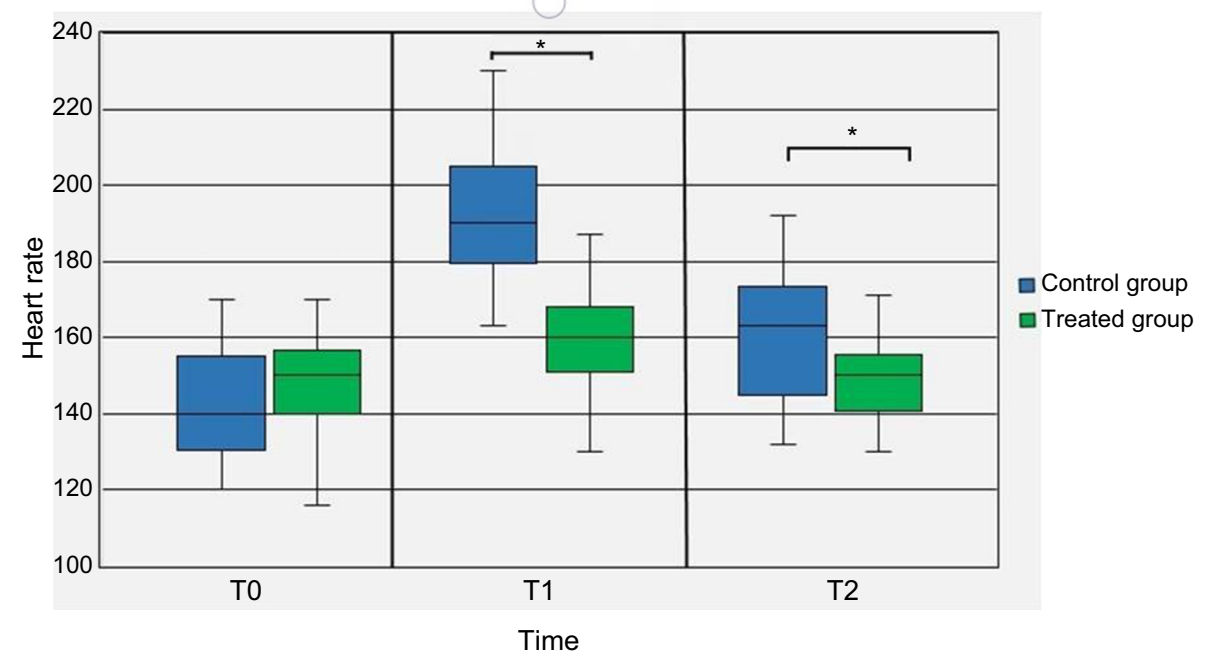

Figure 2 Heart-rate trend from T0 to T2 in control and treated groups. $* P<0.05$. 


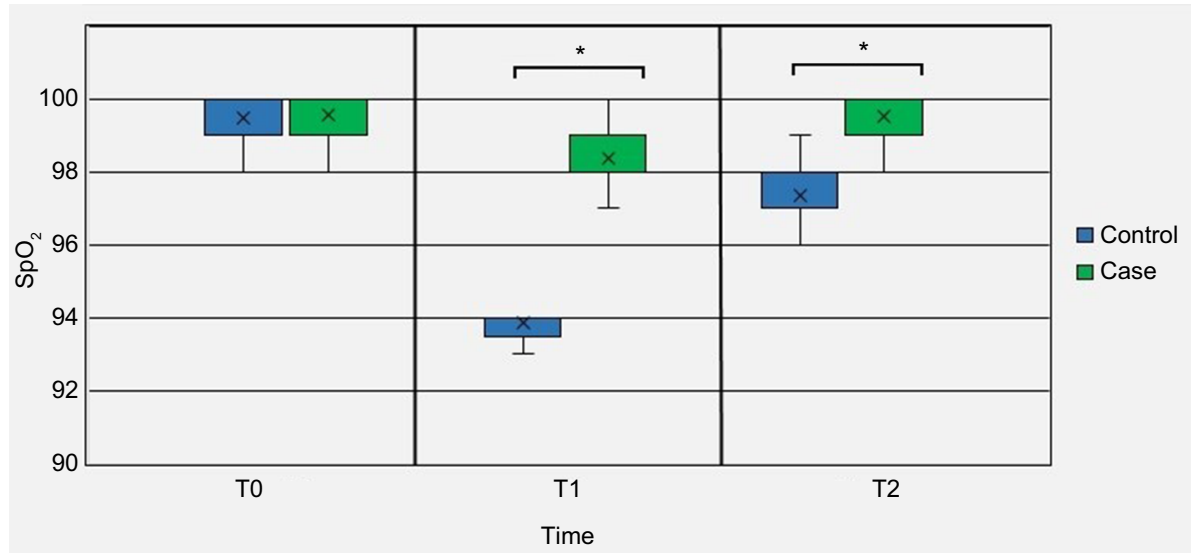

Figure $3 \mathrm{SpO}_{2}$ trend from T0 to T2 in control and treated groups. $* P<0.05$.

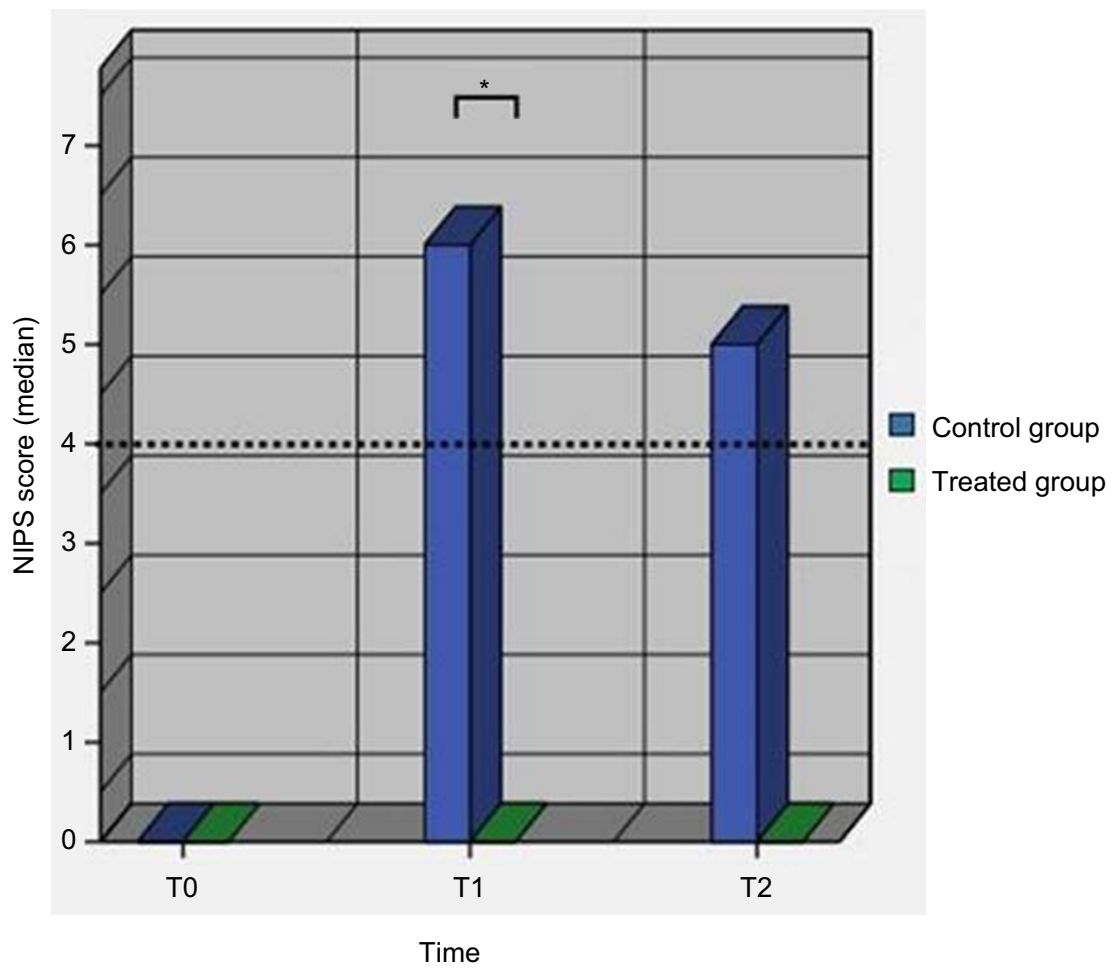

Figure 4 Neonatal Infant Pain Scale (NIPS) scores from T0 to T2 in control group and treated group.

Notes: Dashed line points out the threshold of the pain. A score $\geq 4$ indicates pain. $* P<0.05$.

the Neonatal Facial Coding System to assess pain reactions and reported any difference between sucrose administration and breast-feeding on cortical activity measured by NIRS during a painful procedure. Stevens et al reported that sucrose was effective to treat pain associated with a single heel lance in term neonates admitted to tertiary NICUs. ${ }^{10}$ Efe et al studied three random groups (sucrose, breastfeeding, and control) of term newborn infants at 48 hours of life and found similar pain responses in the sucrose and breast-feeding groups using the NIPS. They also reported that newborns given sucrose spent less time crying than breast-fed newborns. ${ }^{16}$

Thakkar et al demonstrated that sucrose in combination with NNS was more effective than sucrose alone in controlling pain in full-term newborns undergoing heel-stick procedures in the first hours of life, ${ }^{3}$ and this finding led us to evaluate its effect in a population of newborns over the first week of life. No studies have reported data regarding the efficacy of $24 \%$ sucrose for analgesia in healthy newborns over the first week of life. 
Our results may represent one of the most important future perspectives in this field, evaluating the efficacy of oral $24 \%$ sucrose for complete analgesia in term infants over time. Few studies have evaluated $\mathrm{HR}$ and $\mathrm{SpO}_{2}$ responses after noxious stimulation in term infants beyond the NICU setting. The use of $\mathrm{HR}$ and $\mathrm{SpO}_{2}$ as outcome measures and the evidence that a proper analgesic procedure can protect patients from pain-related variation in vital parameters represents the second main finding of our paper as these physiological responses to pain objectify recognition and care of pain during unpleasant experiences. Furthermore, the fact that changes in $\mathrm{HR}$ and $\mathrm{SpO}_{2}$ are not significant in infants treated with $24 \%$ sucrose even after 1 minute from venipuncture demonstrates a possible protective effect from repercussions subsequent to the pain stimulus. ${ }^{17}$

Previously, we found that sensorial saturation, a noninvasive, easily performed, and reproducible technique, was effective for analgesia during heel prick in neonates. ${ }^{6}$ During sensorial saturation, mothers simultaneously attract their infant's attention by massaging the cheek, speaking to the baby gently, and administering $1 \mathrm{~mL}$ $10 \%$ oral glucose. ${ }^{6}$ There is evidence that sweet solutions $<18 \%$ are ineffective in reducing infant pain optimally. ${ }^{10}$ In this study, we used $24 \%$ sucrose associated with NNS to control pain, and we verify complete analgesia through a reduction in pain-related variations in $\mathrm{HR}$ and $\mathrm{SpO}_{2}$ in term newborns.

This study has some limitations. It did not include a control group receiving a placebo (sterile water). However, to assign infants randomly to a placebo and deny them a treatment would have been unethical. The small sample size does not allow us to reach conclusive results, but the findings are significant and pave the way to widespread use of sucrose $24 \%$ associated with NNS.

In conclusion, $1 \mathrm{~mL} 24 \%$ oral sucrose with NNS prior to and during venipuncture is a noninvasive, adequately safe, and reproducible tool for complete analgesia in healthy term newborns over the first week of life. It also protects against pain-related variations in $\mathrm{HR}$ and $\mathrm{SpO}_{2}$. Replication of this study in a large trial is needed to implement coverage of pain control in babies. New research will address barriers to scaling up and evaluate $24 \%$ sucrose efficacy immediately after birth at the time of intramuscular injection of vitamin $\mathrm{K}$ for vitamin $\mathrm{K}$ deficiency-related bleeding prophylaxis and beyond the neonatal period.

\section{Acknowledgments}

This study was supported by EURAIBI (EURope Against Infant Brain Injury; Onlus Foundation). The authors had no financial arrangements, nor were there other factors that could compromise the objectivity of the research.

\section{Disclosure}

The authors report no conflicts of interest in this work.

\section{References}

1. Hartley C, Goksan S, Poorun R, et al. The relationship between nociceptive brain activity, spinal reflex withdrawal and behaviour in newborn infants. Sci Rep. 2015;5:12519.

2. Fitzgerald M. What do we really know about newborn infant pain? Exp Physiol. 2015;100(12):1451-1457.

3. Thakkar P, Arora K, Goyal K. To evaluate and compare the efficacy of combined sucrose and non-nutritive sucking for analgesia in newborns undergoing minor painful procedure: a randomized controlled trial. J of Perin. 2015:1-4.

4. Brummelte S, Grunau RE, Chau V, et al. Procedural pain and brain development in premature newborns. Ann Neurol. 2012;71(3):385-396.

5. Perrone S, Bellieni CV, Negro S, et al. Oxidative stress as a physiological pain response in full-term newborns. Oxid Med Cell Longev. 2017;2017:3759287-7.

6. Bellieni CV, Cordelli DM, Marchi S, et al. Sensorial saturation for neonatal analgesia. Clin J Pain. 2007;23(3):219-221.

7. Costa P, Bueno M, Oliva CL, Elci de Castro T, Ponce de Camargo P, Kimura AF. Analgesia and sedation during placement of peripherally inserted central catheters in neonates. Rev Esc Enferm USP. 2013;47(4):801-807.

8. Stevens B, Yamada J, Ohlsson A. Sucrose for analgesia in newborn infants undergoing painful procedures. Cochr Data of Syst Rev. 2016; 7:CD001069.

9. Lee GY, Yamada J, Kyololo O, Shorkey A, Stevens B. Pediatric clinical practice guidelines for acute procedural pain: a systematic review. Pediatrics. 2014;133(3):500-515.

10. Stevens B, Yamada J, Campbell-Yeo M, et al. The minimally effective dose of sucrose for procedural pain relief in neonates: a randomized controlled trial. BMC Pediatr. 2018;18(1):85.

11. Rodríguez RG, Pattini AE, Roberto GR, Andrea EP. Neonatal intensive care unit lighting: update and recommendations. Arch Argent Pediatr. 2016;114(4):361-367.

12. Lago P, Garetti E, Pirelli A. Linee guida per la prevenzione ed il trattamento del dolore nel neonato: Gruppo di Studio di Rianimazione Neonatale, Societ Italiana di Neonatologia; 2016.

13. Lawrence J, Alcock D, McGrath P, Kay J, Macmurray SB, Dulberg C. The development of a tool to assess neonatal pain. Neonatal Netw. 1993;12(6):59-66.

14. Grunau RE, Whitfield MF, Petrie-Thomas J, et al. Neonatal pain, parenting stress and interaction, in relation to cognitive and motor development at 8 and 18 months in preterm infants. Pain. 2009;143(1-2):138-146.

15. Rioualen S, Durier V, Hervé D, Misery L, Sizun J, Roué JM. Cortical pain response of newborn infants to venepuncture: a randomized controlled trial comparing analgesic effects of sucrose versus breastfeeding. Clin J Pain. 2018;34(7):650-656.

16. Efe E, Savaşer S. The effect of two different methods used during peripheral venous blood collection on pain reduction in neonates. Agri. 2007;19(2):49-56.

17. Meesters N, Simons S, van Rosmalen J, Reiss I, van den Anker J, van Dijk M. Waiting 2 minutes after sucrose administration-unnecessary? Arch Dis Child Fetal Neonatal Ed. 2017;102(2):F167-F169. 
The Journal of Pain Research is an international, peer reviewed, open access, online journal that welcomes laboratory and clinical findings in the fields of pain research and the prevention and management of pain. Original research, reviews, symposium reports, hypothesis formation and commentaries are all considered for publication
The manuscript management system is completely online and includes a very quick and fair peer-review system, which is all easy to use. Visit http://www.dovepress.com/testimonials.php to read real quotes from published authors. 